\title{
An interactive platform to guide catheter ablation in human persistent atrial fibrillation using dominant frequency, organization and phase mapping
}

Xin Li, PhD¥*; João L Salinet, PhD†¥; Tiago P Almeida, $\mathrm{PhD}^{*} \dagger$; Frederique J Vanheusden, $\mathrm{PhD}^{* *}$; Gavin S Chu, MB BChir, MA(Cantab), MRCP(UK)‡§; G André Ng, MBChB, PhD, FRCP(Glasg), FRCP, FESC $\ddagger \S ;$ Fernando S Schlindwein, PhD, DSc*\#

*Department of Engineering, University of Leicester, UK; †Biomedical Engineering, Center for Engineering, Modelling and Applied Social Sciences, Universidade Federal do ABC, Brazil; ¥ Bioengineering Division, Heart Institute (InCor), Brasil; **Institute of Sound and Vibration Research, University of Southampton, UK; $\ddagger$ Department of Cardiovascular Science, University of Leicester, UK; §University Hospitals of Leicester NHS Trust, UK; \#National Institute for Health Research Leicester Cardiovascular Biomedical Research Unit, Glenfield Hospital, UK.

Address for correspondence:

Dr. Fernando S. Schlindwein,

Department of Engineering, University of Leicester,

University Road, Leicester, LE1 7RH, England, UK

Email: fss1@leicester.ac.uk

Tel: +44 (0)116252 5053 / Fax: +44 (0)116252 2619 


\section{Abstract}

Background and Objective: Optimal targets for persistent atrial fibrillation (persAF) ablation are still debated. Atrial regions hosting high dominant frequency (HDF) are believed to participate in the initiation and maintenance of persAF and hence are potential targets for ablation, while rotor ablation has shown promising initial results. Currently, no commercially available system offers the capability to automatically identify both these phenomena. This paper describes an integrated 3D software platform combining the mapping of both frequency spectrum and phase from atrial electrograms (AEGs) to help guide persAF ablation in clinical cardiac electrophysiological studies.

Methods: 30 s of 2048 non-contact AEGs (EnSite Array, St. Jude Medical) were collected and analyzed per patient. After QRST removal, the AEGs were divided into $4 \mathrm{~s}$ windows with a $50 \%$ overlap. Fast Fourier transform was used for DF identification. HDF areas were identified as the maximum DF to $0.25 \mathrm{~Hz}$ below that, and their centers of gravity (CGs) were used to track their spatiotemporal movement. Spectral organization measurements were estimated. Hilbert transform was used to calculate instantaneous phase.

Results: The system was successfully used to guide catheter ablation for 10 persAF patients. The mean processing time was $10.4 \pm 1.5 \mathrm{~min}$, which is adequate comparing to the normal electrophysiological (EP) procedure time (120 180 min).

Conclusions: A customized software platform capable of measuring different forms of spatiotemporal $A E G$ analysis was implemented and used in clinical environment to guide persAF ablation. The modular nature of the platform will help electrophysiological studies in understanding of the underlying AF mechanisms.

Keywords: atrial fibrillation, noncontact mapping, frequency mapping, phase mapping, catheter ablation, intracardiac electrogram, diagnostic software 


\section{Introduction}

Atrial fibrillation (AF) is the most common sustained cardiac arrhythmia found in the clinical practice, affecting around $1 \%$ of the general population [1]. AF increases the risk of stroke by fivefold and it is also related with increased mortality and significant costs in medical treatments $[2,3]$. While paroxysmal $\mathrm{AF}(\mathrm{pAF})$ can be effectively treated by radiofrequency catheter ablation, the outcomes for catheter ablation in patients with persistent AF (persAF) remain suboptimal due to limited understanding of its underlying pathophysiological mechanisms $[1,4]$.

Electrophysiological (EP) studies have shown that structural and electrical remodeling in the atrial tissue are induced by sustained AF. The remodeled tissue could generate focal ectopic activities that could propagate and form re-entry circuits. The atrial regions hosting such behaviors are important in the initiation and perpetuation of the arrhythmia $[5,6]$.

Dominant frequency (DF) measured from atrial electrograms (AEGs) is believed to represent their main activation rate [7, 8]. Therefore, AEGs with high DF (HDF) might represent regions with rapid electrical activations and are potential targets for persAF ablation [9]. Additionally, organization index (OI) and regularity index (RI) have been used to estimate the frequency organization of AEGs, which might help in the identification of the underlying activations [8-10].

Phase analysis was introduced as a promising technique to identify rotors and their spatiotemporal propagation that are potential AF drivers [11, 12]. Recent studies have shown beneficial outcomes in persAF ablation by targeting rotors defined by points of singularity in phase maps $[12,13]$. Both HDF and phase mapping might help to identify potential AF drivers and ablation of those areas might contribute to $A F$ treatment $[13,14]$.

Most EP studies consider point-by-point sequential AEG recording. A recent multi-center study concluded that additional high frequency source ablation beyond pulmonary vein 
isolation (PVI) using sequential mapping did not improve the outcome of persAF patients [15].This finding may suggest that simultaneous signal collection should be preferred. For instance, the DF measured from AEGs was shown to be spatiotemporally unstable during persAF, but it might have cyclic reappearance in some atrial regions, suggesting that simultaneous recordings are needed to characterize such regions [16]. Additionally, proper atrial phase mapping can only be achieved with simultaneous atrial recordings [11]. Currently, multi-electrode array (MEA) and baskets provide simultaneous global unipolar AEG measurements [17]. However, there are no commercial solutions for online DF analyses for simultaneous unipolar AEGs.

The main objective of the current study was to design, develop and validate a quasi-real time interactive graphic user interface (GUI), incorporating these multiple parameters (frequency, organization and phase) to guide EP studies in persAF patients.

\section{Computational Methods}

\section{Electrophysiological study and platform validation}

The present study was approved by local ethics committee for patients undergoing AF ablation at the University Hospitals of Leicester NHS Trust. The software platform, named USURP-GUI, was designed for the USURP-AF (Understanding the electrophysiological substrate of persistent atrial fibrillation) study. Ten persAF patients undergoing catheter ablation in the left atrium (LA) for the first time were enrolled. The patients were given anticoagulant drugs to reduce the risk of blood clots before the procedure. Under fluoroscopic guidance, after bilateral femoral venous access was achieved, a quadripolar catheter and a deflectable decapolar catheter were placed at His position and coronary sinus respectively. Trans-septal puncture was performed to gain access to the LA. A highdensity non-contact mapping (NCM) MEA catheter (EnSite Array, St. Jude Medical, USA) and a conventional deflectable mapping catheter were deployed. Anticoagulant drugs 
were administered to maintain an activated clotting time $>300 \mathrm{~s}$. A high-resolution 3D LA geometry was generated using electro-anatomical mapping (EnSite Velocity, St. Jude Medical, USA) and anatomical locations were annotated. 2048 channels of unipolar AEGs (sampling frequency: $2034.5 \mathrm{~Hz}$ ) were simultaneously collected for $30 \mathrm{~s}$, as well as the 12-lead ECG. During the EP procedure, the data were exported from the EnSite Velocity system using a DVD-RW. The data were transferred to a personal computer and investigated on the USURP-GUI. The results were exhibited on a screen available to the electrophysiologist performing the ablation to help identifying the atrial regions of interest. Figure 1 illustrates the steps of using the USURP-GUI to guide catheter ablation during EP studies.

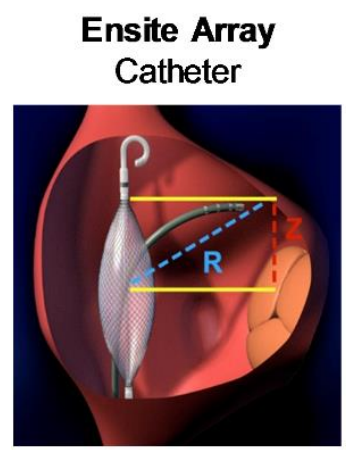

(a)

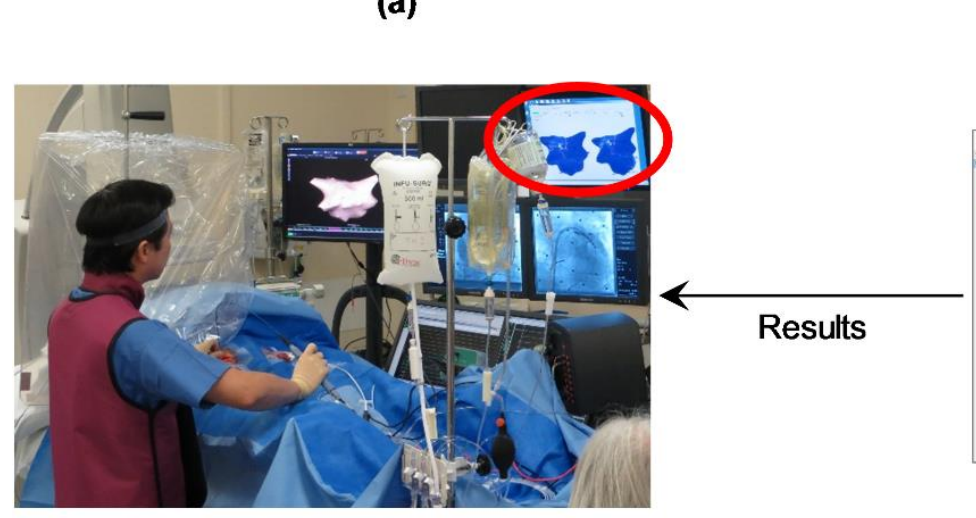

(d) Radiofrequency Ablation Guided by USURP-GUI

\section{Ensite Velocity} Mapping system

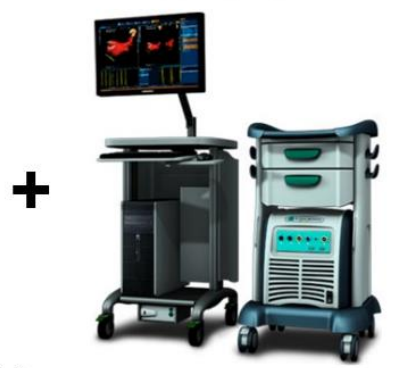

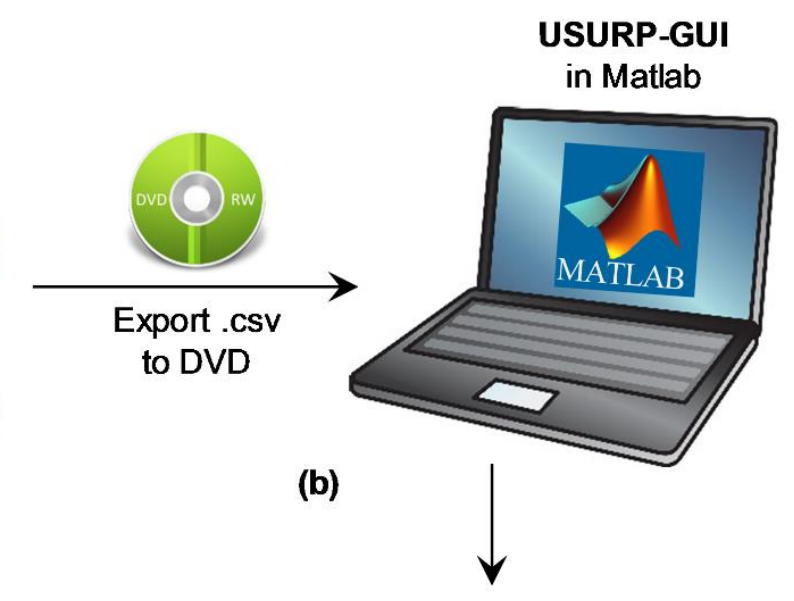

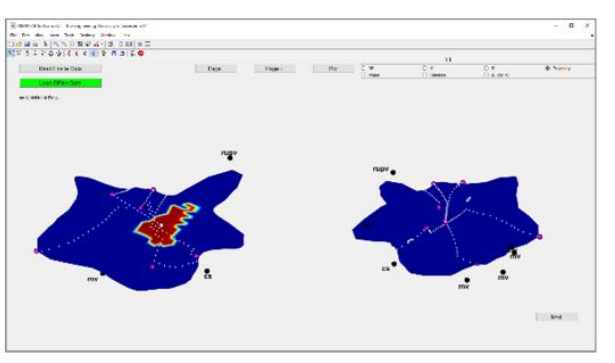

(c) USURP-GUI screen Viewed by the cardiologist performing ablation 
Figure 1. The workflow of using the proposed platform to guide catheter ablation: (a) NCM array and EnSite Velocity system used to collect the data; (b) information exported using DVD-RW disks to laptop running MATLAB; (c) USURP-GUI screen; (d) results exhibited to the cardiologist to help guide ablation.

\section{The USURP-GUI Platform}

The USURP-GUI allows for online and offline EP investigations, by reading either exported comma-separated-value data from EnSite Velocity system (.csv), or loading presaved MATLAB (.mat) files. The simultaneous AEGs and electrocardiograms (ECGs) were exported from the EnSite Velocity system during online analysis, and the processed results were saved for offline studies. Figure 2 shows a schematic representation of the data processing steps of the platform during the online analysis. All the steps are explained further in the subsequent sections. The platform was developed using MATLAB (Mathworks, MA, USA, version 2014a). A laptop running 64 bits Windows 7 professional operating system (Microsoft, Redmond, WA, USA) with Intel i7-4800MQ quad-core processor, 32 GB DDR3 RAM and 32 GB solid-state drive (SSD) was used during the EP procedure. 


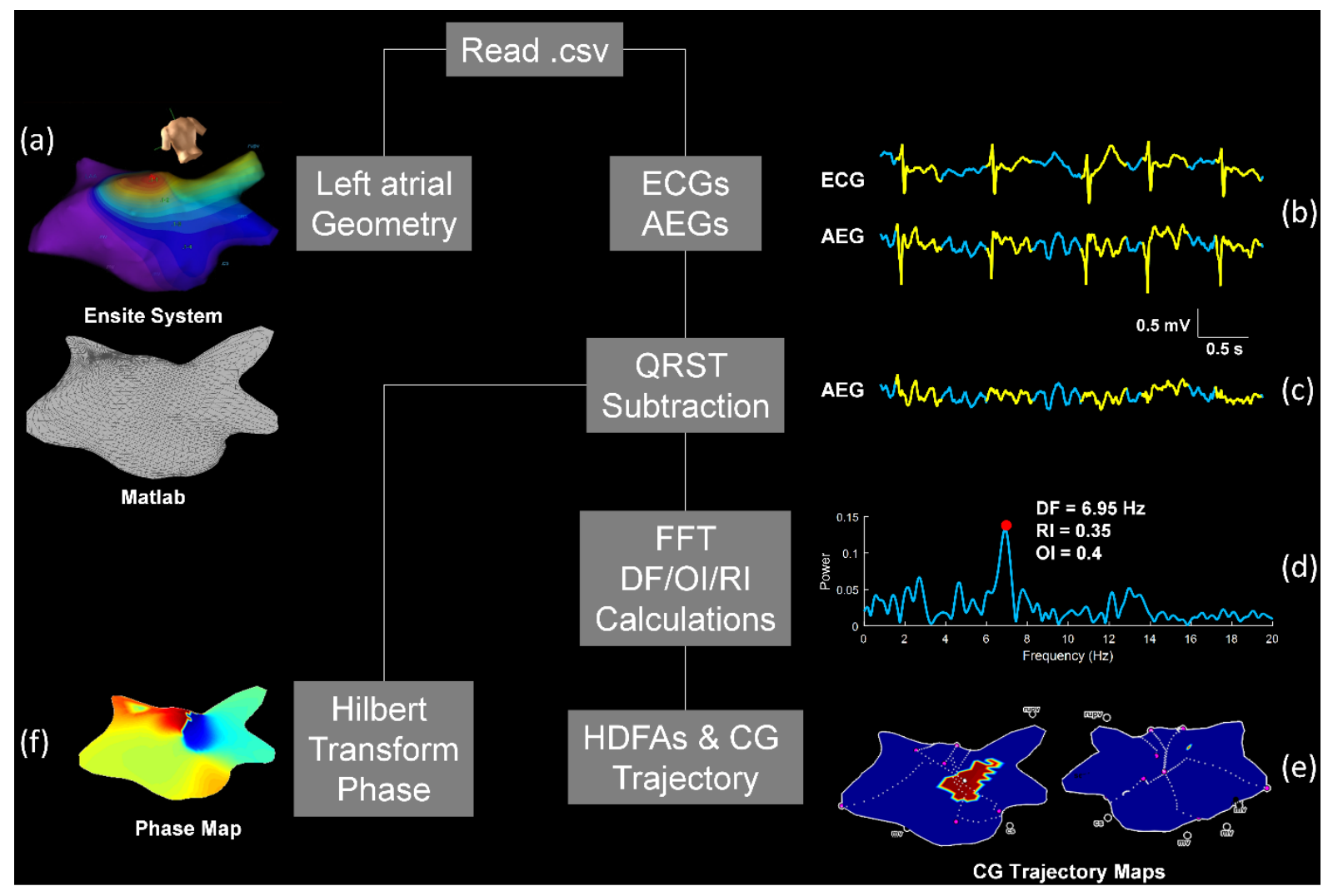

Figure 2. A schematic representation of the data processing procedures of the platform during online analysis: (a-b) The process starts by reading the comma-separated files (.csv) exported from the EnSite Velocity mapping system, and extracting the information needed for the analysis: ECGs, AEGs, 3D atrial mesh geometry and the anatomical labels. (b-c) The QRS onsets and T wave ends are detected from one of the ECG leads (chosen by the user). After that, QRST subtraction is performed to remove the ventricular far-field influence from the AEGs. (d) The DF of the AEG segment is identified, and the $\mathrm{OI}$ and RI calculated. (e) HDF maps are generated for each time window and the centers of gravity (CG) of HDF regions are identified. The trajectories of the CGs are plotted, and sequential CGs are connected with a dotted line to help visualize the movement of HDF areas along time. (f) Phase maps are generated from the AEGs. The processed data are saved and can be re-loaded later for offline analysis. 


\section{QRST subtraction}

Ventricular far-field activity present in the AEGs might appear as misleading frequency components on the atrial frequency spectrum, affecting the accuracy of both the identification of the DF peak and phase mapping [18]. Therefore, a QRST subtraction technique was performed to remove the effect of ventricular far-fields for the 2048 virtual AEGs as previously described [19]. Before the subtraction, the user can select which ECG lead should be used. From this lead, a QRS onset and the end of the T wave are found and the fiducial points are projected to the AEGs after coherent delay alignment. Time average (adaptive) templates of local ventricular influence for each $A E G$ are then removed by subtracting the template. The AEGs after QRST removal were used for further analysis (Figure 2 b-c).

\section{DF Mapping}

The AEGs were divided into $4 \mathrm{~s}$-long segments with $50 \%$ overlap [16]. For each segment, Fast Fourier transform (FFT) was performed to estimate the frequency spectrum (Eq. 1). FFT evaluates the discrete Fourier transform defined as below [20].

$$
X_{k}=\sum_{n=0}^{N-1} x_{n} \cdot e^{\frac{-i 2 \pi k n}{N}} \quad k=0, \ldots, N-1
$$

A Hamming window was used to reduce the amplitude of the side lobes around the DF peak in the power spectrum [21].

A zero padding factor of 5 was applied when performing the FFT, resulting in a frequency step of $0.05 \mathrm{~Hz}$. DF was defined as the frequency peak in the power spectrum within the physiological range of $4-10 \mathrm{~Hz}[16,22]$. 


\section{Assessment of the frequency organization}

Although it is believed that DF correlates with the main activation rate representing the local periodic activities, it is important to investigate the relationship between the DF and the remaining frequency components present in the frequency spectrum of the AEGs [10]. Regularity and organization index have been used to assess the relevance of the DF in the frequency spectrum of $A E G s$, providing an assessment of the $A E G$ activation organization [8-10]. In the present study, RI was defined as the ratio of the area of the DF peak and the total area of the power spectrum (Eq. 2).

$$
R I=\frac{\sum_{f=f_{D F}-k}^{f_{D F^{+k}}} X[f]}{\sum_{f=F_{l}}^{F_{h}} X[f]}
$$

where $f_{D F}$ is the DF of the unipolar AEG segment, $f$ is the frequency, $X[f]$ is the power spectrum, $k$ defines a DF width threshold (here set at $0.375 \mathrm{~Hz}$ ), $F_{l}$ and $F_{h}$ are the lower and higher boundaries of the physiology range of the arrhythmia respectively (in our case $4 \mathrm{~Hz}$ to $10 \mathrm{~Hz}$ ).

Similarly, OI is defined as the ratio of the area of the DF peak and its harmonics, and the total area of the power spectrum (Eq. 3) [2].

$$
O I=\frac{\sum_{f=f_{D F}-k}^{f_{D F^{+}}} X[f]+\sum_{n=1}^{N} \sum_{f=h_{n}-k}^{h_{n}+k} X[f]}{\sum_{f=F_{l}}^{F_{h}} X[f]}
$$

Where $h_{n}$ are the harmonic peaks of the DF, and $N$ is the total number of harmonic peaks within a certain frequency band. To maintain clinical relevant results, harmonics up to 20 $\mathrm{Hz}$ are included in the calculation.

\section{HDF cloud and the trajectory of CGs}

To track the spatiotemporal behavior of the high frequency regions in the atrium, HDF areas (HDFA) were defined as regions with DF value equal to or higher than the maximum 
DF- $0.25 \mathrm{~Hz}$ for each time window [16] (Eq. 4), where $i$ denotes the electrode number and $n$ is the time window number.

$$
H D F(i, n)=\left\{\begin{aligned}
D F(i, n), & D F(i, n) \geq \max (D F(n))-0.25 \\
0, & \text { otherwise }
\end{aligned}\right. \text { Eq.4 }
$$

The CG of each HDFA was estimated from a 2D rectangular uniform mesh representation in order to summarize the dynamic movement of the sequential HDFAs. For sequential frames, the trajectories of the shortest path between the CGs were also identified using Dijkstra's algorithm on the 3D triangular mesh [23].

\section{Phase mapping}

Hilbert transform $h(t)$ was used to generate an analytic signal $F(t)$, from which the instantaneous phase $\varphi(t)$ of the AEGs can be calculated as the four-quadrant inverse tangent of the ratio of the imaginary $h(t)$ and real part $f(t)$ of the analytic signal (Eq. 5), where $h(t)$ is the Hilbert transform of the original signal $f(t)[11,24]$.

$$
\begin{gathered}
F(t)=f(t)+j h(t)=A(t) e^{j \varphi(t)} \\
\varphi(t)=\tan ^{-1}\left\lfloor\frac{h(t)}{f(t)}\right\rfloor
\end{gathered}
$$

\section{Mesh Triangulation}

The locations of 2048 (64 x 32) virtual electrodes provided by the EnSite Velocity system can be easily manipulated to form a mesh of rectangles. Nevertheless, this type of mesh would result in steep CG trajectories. The atrium was therefore re-meshed to obtain a triangular structure in order to obtain smoother CG trajectories [25].

\section{AEG Visualization}

Considering the importance of visualizing AEGs in atrial regions of interest during EP studies, the algorithm to detect the intersection of triangle plane and the line through the 
current cursor location was implemented based on Cramer's rule [26]. Therefore, the AEG at any node could be visualized.

\section{Saving data for offline analysis}

The processed data, which includes the AEGs after QRST subtraction, frequency measurements, phase, CGs and trajectories, labels and positions of LA anatomical structures, vertices and triangulations of the LA 3D geometry, were saved for retrospective offline analysis.

\section{Processing time}

In order to test the feasibility of the platform during EP procedures, the processing time for $30 \mathrm{~s}$-long segments from five patients was tested in a 'dry run'. The processing time was assessed for five modules, accordingly: reading exported data; QRST subtraction; frequency calculations; phase calculation; and saving results. The processing time for each module was measured within MATLAB.

\section{System Description}

The proposed platform was developed using MATLAB (Mathworks, MA, USA, version 2014a), including a user-oriented interface (graphic user interface [GUI]) which can be easily operated by the clinician, providing online and offline analysis of both frequency and phase mapping.

The USURP-GUI has embedded the following main features: Read/load data; map type selection; time window selection; rotation control.

\section{Read or load the data}

The 'Read/Load' buttons on the left upper corner of the main window are used for selecting the type of analysis (Figure 3). If the user clicks on one of the buttons, a file 
selection window will pop up. The 'Read' button is used for online investigations. The user is expected to select 3 files (.csv), named Labels, ECG and AEGs. From the EnSite Velocity system, 2048 virtual unipolar electrograms were exported with the filename 'Virtuals_Grid_2048.csv'. This .csv file contains both atrial electrograms and the 3D locations of the virtual electrodes. With the scattered 3D coordinates, the left atrial geometry with 2048 virtual electrodes were reconstructed. Additional, the surface ECGs ('ECG_FILTERED.csv') and anatomical labels ('Labels.csv') were also exported and processed by the platform. The 'Load' button is used for offline analyses. In this case, only one MATLAB file (.mat) containing all the data is selected. For both cases, once the data files are selected, the button changes to red showing that the tool is processing the data and will turn green when the system is ready to show the results. 


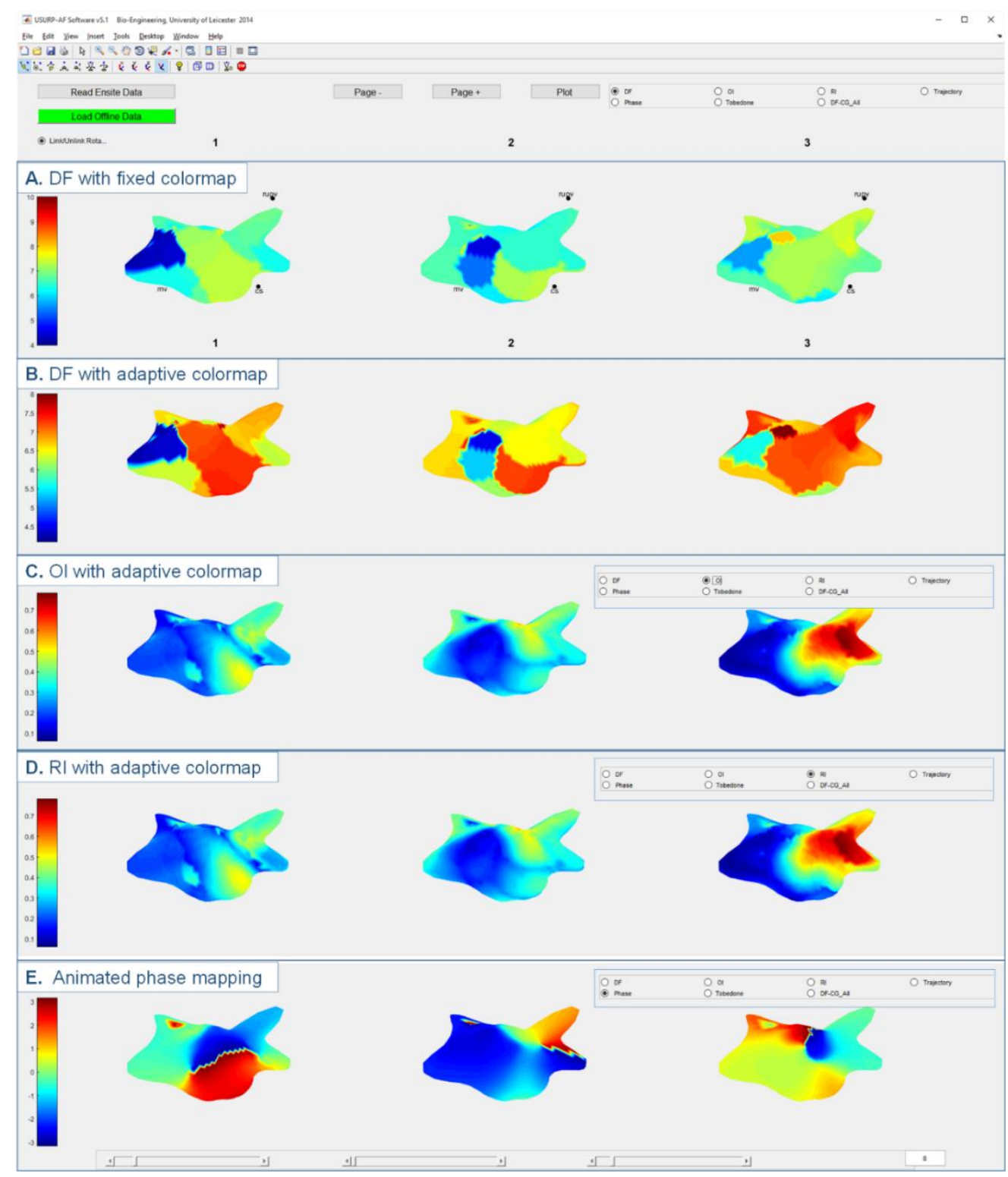

Figure 3. Illustration of the USURP-GUI features and interface. A. DF with fixed color map B.DF with adaptive color map. C. OI with adaptive color map D. RI with adaptive color map E. Phase maps with slider to play, pause, forward and backward. 


\section{Map type panel}

On the right upper corner of the main page (Figure 3), a group of buttons allows the user to select the type of $A E G$ analysis to be projected in the platform on the 3D mesh. The AEG analysis includes DF maps, OI, RI, phase maps, trajectory and a summary of CGs.

\section{DF maps}

The default analysis is 'DF', in which a group of two by three LA maps is generated with the DF values projected in the LA geometry (Figure 3A and 3B). The first row with three maps (Figure $3 \mathrm{~A}$ ) represents sequential windows of DF with the color code range fixed in 4-10 Hz, while the second row (Figure 3B) shows the same maps, but using a different frequency range, adjusted from the minimum to the maximum DF values within the three maps. This provides a more detailed look of the landscapes and contours of the DF maps.

\section{Ol and RI maps}

If the buttons $\mathrm{OI}$ or $\mathrm{RI}$ are selected, the three maps in the first row will continue to show the DF values, but the three maps in the second row will change to either OI or RI according to the selection - of the same time window (Figure $3 \mathrm{C}-\mathrm{D}$ ). This allows the users to investigate the DF maps with the frequency spectrum organization maps simultaneously, for each time window (Figures 3C and 3D).

\section{Phase maps}

Similarly, the phase calculated from the AEGs can be projected in the LA geometry on the maps in the second row (Figure 3E). Whilst frequency features are measured over 4 $s$ time windows, the phase is instantaneous and hence measured at each time frame. Therefore, a slider was designed to assess the sequential frames of the phase in the 3D LA maps. Additionally, the frame step can be set by the user in order to control the play speed by entering an integer 'step' number in the right lower corner. 


\section{Trajectory of CG maps}

Once the 'Trajectory' button is selected, two large maps showing the HDF cloud and its CG are displayed, as measured from the first time window (Figure 4A). The 'Next' button updates the maps with the HDF and CG measured from the next time window, and the trajectory of the CGs on the 3D mesh is also projected on the map, as illustrated in Figure 4A. In this case, the red area refers to the HDF cloud measured from the selected time window, and its respective $C G$ is represented as the white dot with black edge (inside the cloud). The magenta dots represent the CGs measured from previous time windows, and the white dotted line is the shortest path between consecutive CGs projected on the mesh. 


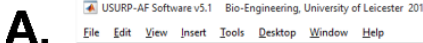

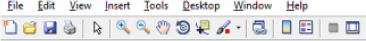

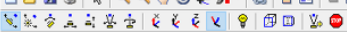

Read Ensite Data

Load Offine Data

Page. -

Plot Oof

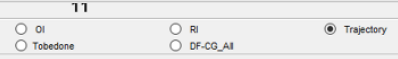

- LinkUnilinkRota
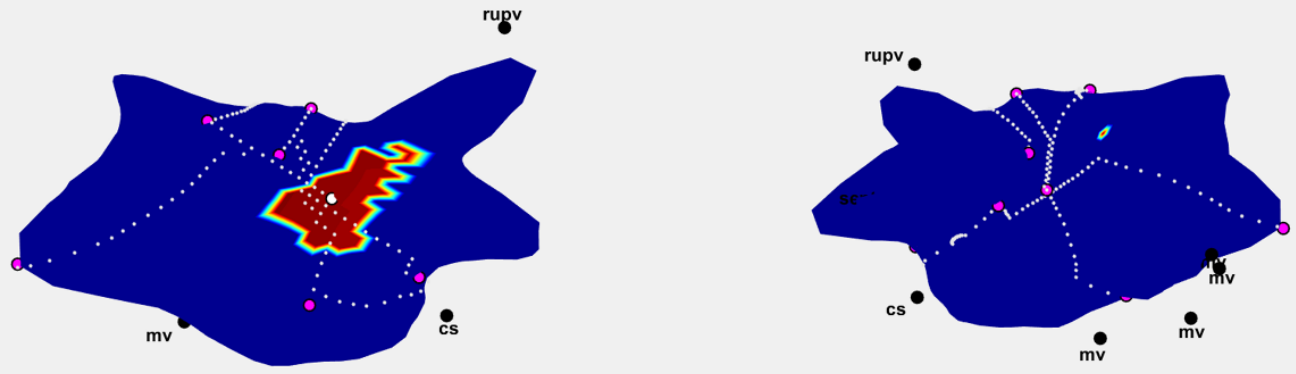

B.

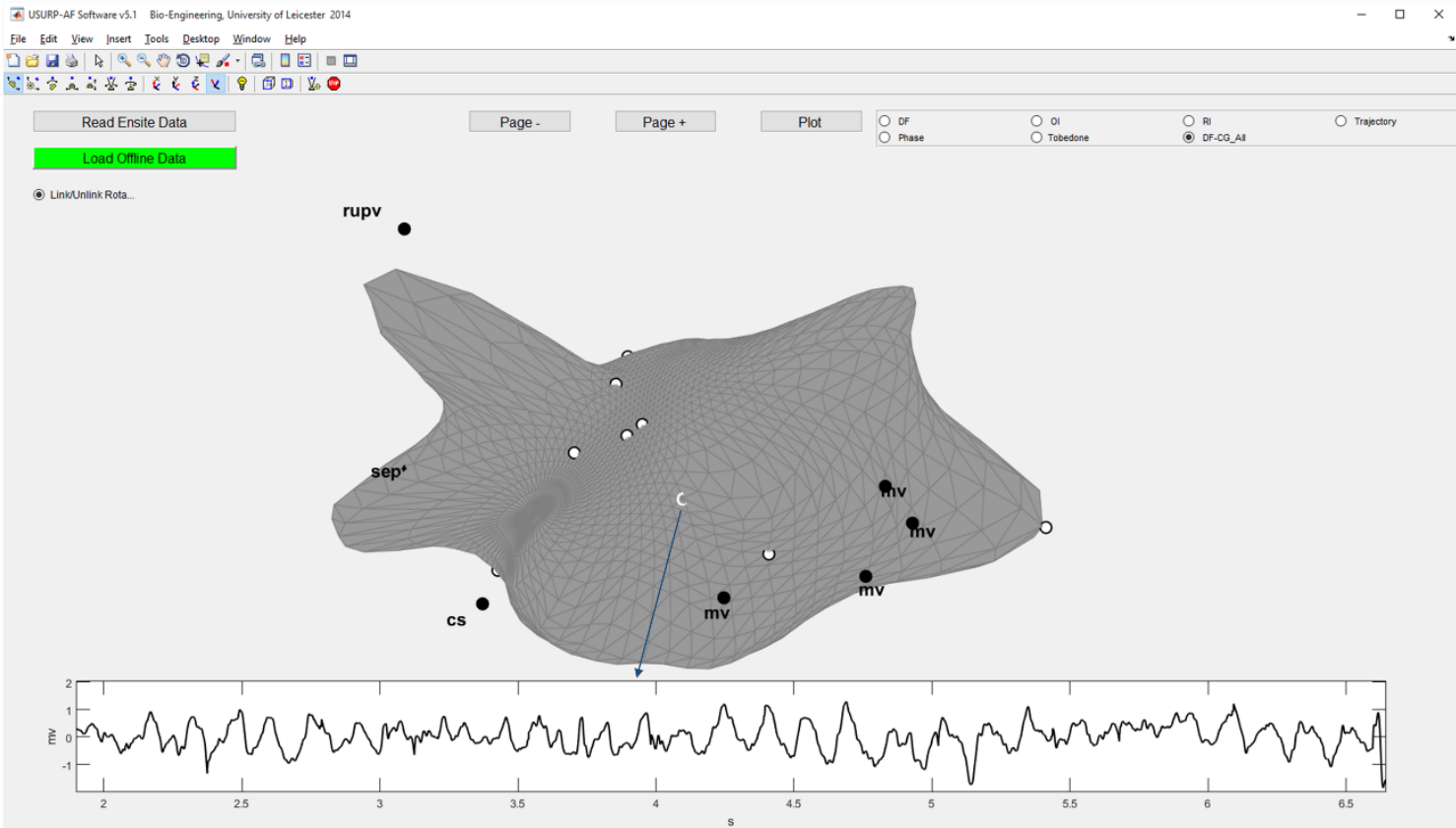

Figure 4. A. CGs and the trajectories between sequential CGs (current HDFA in red zone; previous CGs in magenta circles; current CG in white circle; trajectories tracking the 
movement of CGs in white dotted lines). B. Summary of all the CGs (full white dots) and the AEG of a point selected by the user.

\section{Summary of CGs}

The tool allows to shown one full screen map with the CGs projected on the mesh as measured from all time windows, as a summary of the CGs clustering (Figure 4B). In this analysis, virtual AEGs can be visualized by selecting the nodes on the mesh. The CGs are represented as white dots on a grey 3D LA map.

\section{Rotation Control and Labels}

The 'Link/Unlink' button on the left upper corner (below the 'Load' button) allows linking and unlinking the rotation of the six maps (Figure 3). Thus, the user can rotate the plots simultaneously or individually by selecting and deselecting the button. As seen in Figures 3 to 4 , the software also allows the display of the anatomic references created by the EnSite Velocity system.

\section{Processing Time}

The overall USURP-GUI processing time (mean \pm SD) when pressing the 'Read' button, i.e. during online EP studies, was $624 \pm 90 \mathrm{~s}$ for AEGs with $30 \mathrm{~s}$. The processing time for each module is shown in Figure 5, divided accordingly: reading exported data (204.1 \pm $7.5 \mathrm{~s}$ ); QRST subtraction (220.9 $\pm 97 \mathrm{~s}$ ); frequency calculations (43.9 $\pm 0.2 \mathrm{~s}$ ); phase calculation (130.8 $\pm 4.9 \mathrm{~s})$; and saving results (21.4 $\pm 0.1 \mathrm{~s})$. 


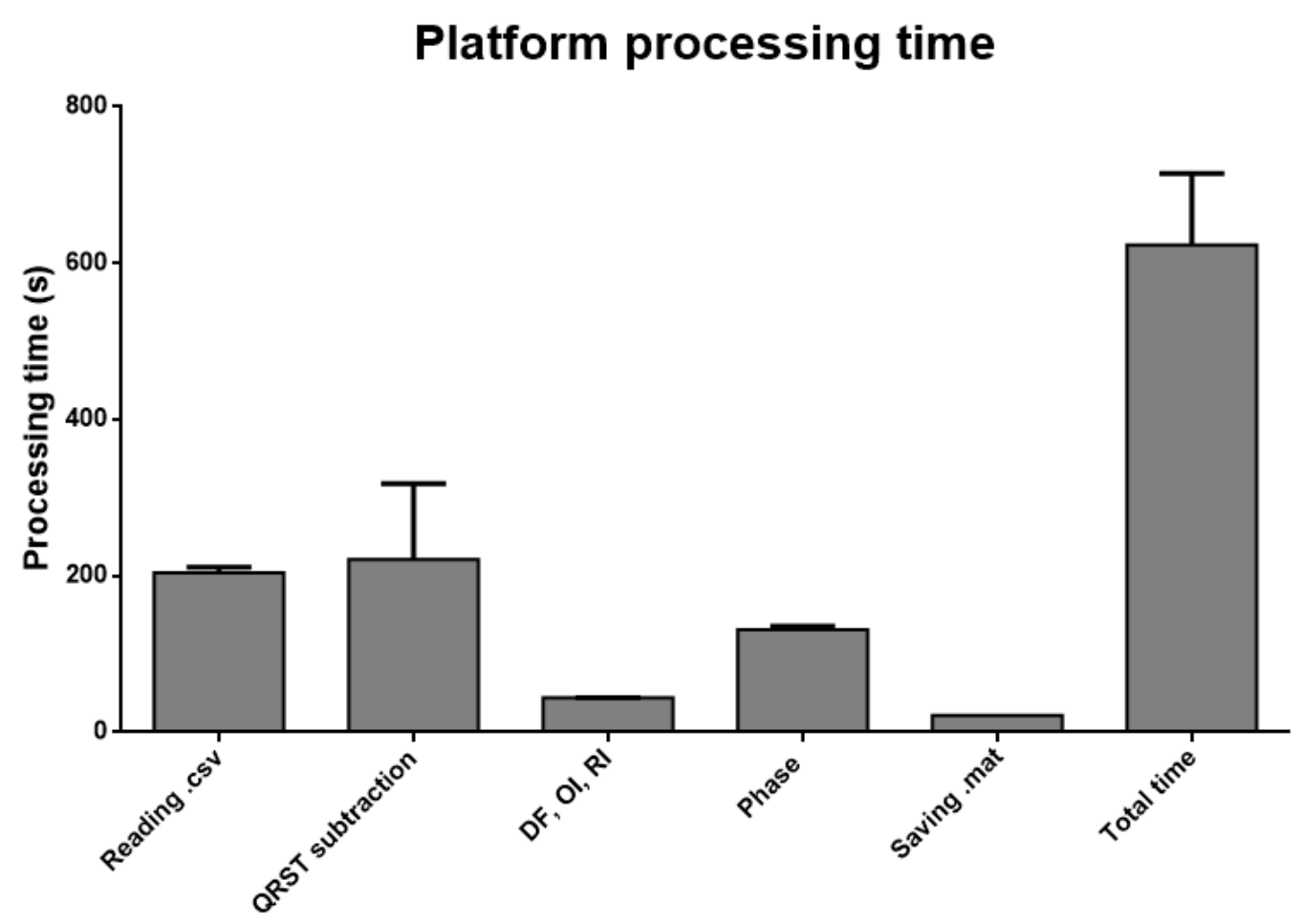

Figure 5 Processing time and standard deviation (bars) of the key modules embedded in USURP-GUI.

\section{Discussion}

Different features measured from AEGs were used individually in previous EP studies, with varying outcomes $[12,13,16,27,28]$. A software platform able to integrate these different $A E G$ features would help to improve our understanding regarding the underlying mechanisms of persAF maintenance and in the identification of targets for ablation.

In the present work, we developed and validated the first software platform that investigates multiple forms of spatiotemporal AEG features, such as frequency and phase analysis that can be used during the EP procedure, to help guide catheter ablation in persAF patients [29]. 


\section{Mechanisms for persAF maintenance}

AF initiation and perpetuation requires both triggers for its onset and a substrate for its perpetuation [30]. In general, patients with pAF are likely to have a predominance of factors that trigger $A F$, whereas patients with persAF are more likely to have a predominance of perpetuating factors due to atrial remodeling [31]. There is, however, overlap between these mechanisms in a sense that patients with pAF might not present identifiable triggers, as well as triggers can be found as the main cause for maintenance of $A F$ in patients with persAF [32].

Catheter ablation has proven to be an effective therapy for $\mathrm{pAF}[33,34]$. As persAF is a more complex arrhythmia than pAF, the identification of atrial areas for successful ablation in patients with persAF remains a challenge due to the co-existence of multiple arrhythmogenic mechanisms [4, 35]. Previous works have shown that the DF measured from AEGs correlates with the local main activation wave during persAF $[9,14,36]$. Therefore, tracking the spatiotemporal behavior of atrial sites hosting HDF could help in the identification of arrhythmogenic regions [16]. Some researchers have argued that HDF might also be caused by wavefront collisions, and OI could help to distinguish AF drivers [37]. In addition, rotor-guided ablation has shown promising success rates in early data $[11,24,38]$. Therefore, the proposed platform would help to understand the relationship between multiple features and study the underlying mechanisms of AF.

\section{Estimating DF using NCM}

Previous studies showed imperfect correlations between the NCM and contact mapping [39-41]47. Schilling et al. found a correlation of $0.74 \pm 0.19$ for 3600 electrograms tested in the right atrium [39]. Later in 2006, Earley et al. showed the similar correlation 0.81 (0.27 to 0.98 ) from the left atrium [40]. Jarman et al. also showed a correlation of $0.7 \pm 0.15$ for 62 random locations in the left atrium [41]. It was also shown that correlation decreased with increasing distance between the endocardial node and the balloon [40, 
42]. However, these comparisons were limited on the correlation of the morphology of electrograms. In the interesting work by Gojraty et al., instead of comparing electrograms in time domain, DFs measured with contact bipolar recordings and noncontact unipolar were compared. Although low correlation was also found between contact and concontact electrograms, there was no significant difference in the mean DFs between contact bipolar and noncontact unipolar signals [43]. Therefore, it is feasible to estimate DFs using noncontact mapping.

\section{FFT Window Size}

The technique of analysing DF using non-contact mapping of virtual AF electrograms has previously been studied and validated by other researchers [16, 43, 44]. Sanders and colleagues terminated human AF in catheter ablation guided by contact DF mapping using windows of 4096 samples with sampling frequency of $1 \mathrm{kHz}$, using time windows of 4 seconds [9]. $\mathrm{Ng}$ and colleagues showed relatively good correlation between DF calculated using 2 seconds time sub-widow and full 4 seconds window, and 4 consecutive windows of 4 seconds could improve the correlation of DF with the atrial activation rate comparing to the DF calculate using just a single window [45-47]. Later, Stiles et al. studied the correlation between the DF using AF EGMs from 1 to 7 second time windows and the DF from 8 second window, and they concluded that EGMs with duration of 5 seconds or longer could accurately describe the DF [48].

In the current study, 4-second windows were chosen as it has been shown by other investigators that if smaller "slide windows" were chosen, the electrogram window did not accurately characterize DF sites [3, 9, 48]. In addition, longer FFT window segments would contribute to a considerable increase in the appearance of stability of DF over time, which could be potentially misleading the localization of potential AF sites [16]. In the spectral analysis strategy, an anti-leakage window has been implemented to reduce undesirable effects due to abrupt discontinuities at the beginning or the end of the 
segment [49]. By considering that the AF signal might have non-stationary components, relevant AF events can occur in periodic intervals that might well be important to the analysis. These events can occur at the beginning or end of the AF window segments using an overlap processing ( $50 \%$ used in this case) would capture the events of interest because with the overlapping of the time records used to calculate the spectrum, the Hamming window function would align with the events thus including them in the calculated spectrum.

\section{Online analysis during EP procedure}

Our results show that the overall processing time (about $10.5 \mathrm{~min}$ ) of the USURP-GUI for 30 s segments of the 2048 channels of AEGs is relatively short compared to the total time of catheter ablation procedures for complex persAF (typically 120-180 min) [50]. It is possible for integrated analysis considering multiple features measured from AEGs during persAF, which would provide a multidimensional perspective of the arrhythmia, in which different aspects of the underlying mechanisms for persAF maintenance would be simultaneously investigated, and may further contribute to improve the efficacy of persAF therapy [51].

\section{Clinical Significance}

Previous studies have shown that the DF of individual AEGs is temporally unstable in persAF, which highlights a serious limitation of the point-by-point sequential mapping technique $[7,16]$. Simultaneous unipolar AEGs can be collected using high-density NCM [17]. DF analysis of such simultaneous unipolar atrial signals during persAF could help to reveal the spatiotemporal distribution of the atrial activations. However, current commercial mapping systems do not provide online DF analysis of the simultaneous unipolar signals. In addition, the USURP-GUI was implemented with advanced spatiotemporal analysis of movement of HDF regions [16]. Even though analysis in the current platform (DF, RI, OI, phase mapping) has been already well established, such 
multiple features are still lacking from available systems, especially using high density NCM.

Commercial systems and research-orientated platforms for the investigation of rotor analysis and complex fractionated atrial electrograms (CFAEs) are usually either builtinto the commercial mapping systems or by reading the exported data from other recording systems [12, 13, 52]. For instance, the RhythmView ${ }^{\circledR}$ mapping system (Topera, Menlo Park, CA, USA) is used to create AF propagation maps using electrograms exported from commonly-used physiological recorder (Bard, Lowell, MA) via USB storages, and the proposed system operates in such similar fashion during the EP procedures.

The present platform was developed as a versatile tool and can be easily expanded to include other algorithms considering different theories of persAF maintenance and arrhythmias, such as CFAEs to further investigate the multiple wavelet hypothesis [52]. Additionally, other tools can be integrated in the USURP-GUI to allow more comprehensive studies of multiple aspects of the arrhythmia, such as HDF histogram mapping to investigate the DF spatiotemporal behaviors [53], recurrent patterns of HDF algorithms [54] and up-to-date classification techniques for CFAE [55]. The preliminary outcome of using this platform was reported [56].

\section{Limitations}

As the current platform runs on MATLAB environment, the data exported from the mapping system were transferred on to the laptop using DVD-RW disks. Clearly, the additional time of burning the DVD and importing data to the USURP-GUI from the DVD has to be considered during the EP. Even though the latest mapping system is able to support USB storages that are much faster comparing to burning DVDs, it would still be interesting to integrate the analysis performed in our platform to the such mapping 
systems themselves, in order to speed up the current ablation guided by additional features.

\section{Conclusions}

We have designed a bespoke software platform capable of handling multiple forms of spatiotemporal AEG analysis, and objectively demonstrated the feasibility of utilizing the platform in a clinical environment during persAF ablation. The proposed mapping platform, USURP-GUI, is a fully automated software interface that provides a 3D representation of the atrium. It can be used to guide persAF ablation considering multiple features measured from AEGs during pers AF - such as the DF and phase - and can be easily expanded to include additional features. The USURP-GUI provides a multidimensional perspective of the arrhythmia, in which different aspects of the underlying mechanisms for persAF maintenance can be investigated, and may further contribute to improve the efficacy of persAF therapy.

\section{Conflicts of Interest Statement}

None to declare.

\section{Acknowledgements and disclosures}

The research leading to these results was funded by the Leicester NIHR Cardiovascular Biomedical Research Unit, UK. Dr. Salinet has received research grants from Conselho Nacional de Desenvolvimento Científico e Tecnológico (CNPq, Brazil, grant $\mathrm{N}$. 200598/2009-0). Dr. Almeida has received research grants from CNPq (N. 200251/20120 ) and Coordenação de Aperfeiçoamento de Pessoal de Nível Superior (Capes). Dr. Vanheusden has received a research grant from NIHR Cardiovascular Biomedical Research Unit, UK and the Engineering and Physical Sciences Research Council (EPSRC). Dr. Chu has received a research grant from St. Jude Medical and honoraria 
from Biosense Webster. Prof. $\mathrm{Ng}$ has received research fellowship from St. Jude Medical and speaker fees and honoraria from Biosense Webster. All other authors have reported that they have no disclosures relevant to the contents of this paper.

\section{Biography}

Dr. Xin Li obtained B.Sc. in Electrical Information Engineering from the University of Science and Technology Beijing 2011, and M.Sc. in Electrical Electronic Engineering from the University of Leeds 2012. He has recently been awarded his Ph.D. from the University of Leicester. He obtained his Ph.D. at the University of Leicester, and is currently a postdoctoral researcher at University of Leicester.

Dr. João L. Salinet obtained an Electrical Engineering degree in 2002 from the Federal University of Santa Catarina and M.Sc. in Biomedical Engineering from the University of Campinas in 2009. He obtained his Ph.D. from the University of Leicester in 2013. Currently, Dr Salinet is a lecturer in Biomedical Engineering at Federal ABC University and Research Associated at the Heart Institute (InCor). His research interests include heart conduction system and cardiac arrhythmias mechanisms on both human and experimental models.

Dr. Tiago P. Almeida obtained a B.E. in Electrical Engineering at São Paulo State University in 2007 and M.Sc. in Biomedical Engineering at the University of Campinas in 2012. He obtained his Ph.D. at the University of Leicester, and is currently a postdoctoral researcher at Federal ABC University.

Dr. Frederique J. Vanheusden obtained B.Sc. and M.Sc. in Biomedicine at Hasselt University and M.Sc. in Molecular Medicine at Cranfield University. He obtained his Ph.D. in Biomedical Engineering at the University of Leicester. He is currently a research fellow at the University of Southampton. 
Dr. Gavin S. Chu graduated from the University of Cambridge in 2004. He is a Specialty Registrar in Cardiology and a Clinical Research Fellow in Cardiac Electrophysiology focusing on translational bioengineering in atrial fibrillation.

Prof. G. André Ng studied Medicine at the University of Glasgow where he obtained his MBChB and Ph.D. He was appointed as Senior Lecturer and Consultant Cardiologist and Electrophysiologist at the University Hospitals of Leicester in 2002, and was awarded Personal Chair of Cardiac Electrophysiology at the University of Leicester in 2013. He leads a team of talented researchers spanning preclinical, clinical, and biomedical engineering areas focused on cardiac arrhythmias and electrophysiology

Dr. Fernando S. Schlindwein obtained an Electrical Engineering degree in 1979 from the Federal University of Rio Grande do Sul, an M.Sc. in Biomedical Engineering from the Federal University of Rio de Janeiro, a Ph.D. in Biomedical Engineering from the Department of Surgery of the University of Leicester in 1990, and a D.Sc. in Biomedical Engineering from the Federal University of Rio de Janeiro in 1992. He joined the Department of Engineering at the University of Leicester as a lecturer in 1992. His main research interests are real-time signal processing and cardiac arrhythmias, especially the characterization and treatment for atrial fibrillation. 


\section{References}

[1] S. Nattel, "New ideas about atrial fibrillation 50 years on," Nature, vol. 415, pp. 219-26, Jan 102002.

[2] J. A. Cairns and S. J. Connolly, "Nonrheumatic atrial fibrillation. Risk of stroke and role of antithrombotic therapy," Circulation, vol. 84, pp. 469-81, Aug 1991.

[3] T. H. t. Everett, L. C. Kok, R. H. Vaughn, J. R. Moorman, and D. E. Haines, "Frequency domain algorithm for quantifying atrial fibrillation organization to increase defibrillation efficacy," IEEE Trans Biomed Eng, vol. 48, pp. 969-78, Sep 2001.

[4] S. Nattel, "Atrial electrophysiology and mechanisms of atrial fibrillation," J Cardiovasc Pharmacol Ther, vol. 8 Suppl 1, pp. S5-11, Jun 2003.

[5] A. Goette, C. Honeycutt, and J. J. Langberg, "Electrical remodeling in atrial fibrillation. Time course and mechanisms," Circulation, vol. 94, pp. 2968-74, Dec 011996.

[6] A. M. Pertsov, J. M. Davidenko, R. Salomonsz, W. T. Baxter, and J. Jalife, "Spiral waves of excitation underlie reentrant activity in isolated cardiac muscle," Circ Res, vol. 72, pp. 631-50, Mar 1993.

[7] J. W. Jarman, T. Wong, P. Kojodjojo, H. Spohr, J. E. Davies, M. Roughton, et al., "Spatiotemporal behavior of high dominant frequency during paroxysmal and persistent atrial fibrillation in the human left atrium," Circ Arrhythm Electrophysiol, vol. 5, pp. 6508, Aug 012012.

[8] J. Tuan, F. Osman, M. Jeilan, S. Kundu, R. Mantravadi, P. J. Stafford, et al., "Increase in organization index predicts atrial fibrillation termination with flecainide post-ablation: spectral analysis of intracardiac electrograms," Europace, vol. 12, pp. 488-93, Apr 2010.

[9] P. Sanders, O. Berenfeld, M. Hocini, P. Jais, R. Vaidyanathan, L. F. Hsu, et al., "Spectral analysis identifies sites of high-frequency activity maintaining atrial fibrillation in humans," Circulation, vol. 112, pp. 789-97, Aug 092005. 
[10] A. C. Skanes, R. Mandapati, O. Berenfeld, J. M. Davidenko, and J. Jalife, "Spatiotemporal periodicity during atrial fibrillation in the isolated sheep heart," Circulation, vol. 98, pp. 1236-48, Sep 221998.

[11] K. Umapathy, K. Nair, S. Masse, S. Krishnan, J. Rogers, M. P. Nash, et al., "Phase mapping of cardiac fibrillation," Circ Arrhythm Electrophysiol, vol. 3, pp. 105-14, Feb 2010.

[12] S. M. Narayan, D. E. Krummen, K. Shivkumar, P. Clopton, W. J. Rappel, and J. M. Miller, "Treatment of atrial fibrillation by the ablation of localized sources: CONFIRM (Conventional Ablation for Atrial Fibrillation With or Without Focal Impulse and Rotor Modulation) trial," J Am Coll Cardiol, vol. 60, pp. 628-36, Aug 142012.

[13] S. M. Narayan, T. Baykaner, P. Clopton, A. Schricker, G. G. Lalani, D. E. Krummen, et $a l .$, "Ablation of rotor and focal sources reduces late recurrence of atrial fibrillation compared with trigger ablation alone: extended follow-up of the CONFIRM trial (Conventional Ablation for Atrial Fibrillation With or Without Focal Impulse and Rotor Modulation)," J Am Coll Cardiol, vol. 63, pp. 1761-8, May 062014.

[14] F. Atienza, J. Almendral, J. Jalife, S. Zlochiver, R. Ploutz-Snyder, E. G. Torrecilla, et al., "Real-time dominant frequency mapping and ablation of dominant frequency sites in atrial fibrillation with left-to-right frequency gradients predicts long-term maintenance of sinus rhythm," Heart Rhythm, vol. 6, pp. 33-40, Jan 2009.

[15] F. Atienza, J. Almendral, J. M. Ormaetxe, A. Moya, J. D. Martinez-Alday, A. HernandezMadrid, et al., "Comparison of radiofrequency catheter ablation of drivers and circumferential pulmonary vein isolation in atrial fibrillation: a noninferiority randomized multicenter RADAR-AF trial," J Am Coll Cardiol, vol. 64, pp. 2455-67, Dec 162014.

[16] J. L. Salinet, J. H. Tuan, A. J. Sandilands, P. J. Stafford, F. S. Schlindwein, and G. A. Ng, "Distinctive patterns of dominant frequency trajectory behavior in drug-refractory persistent atrial fibrillation: preliminary characterization of spatiotemporal instability," $J$ Cardiovasc Electrophysiol, vol. 25, pp. 371-9, Apr 2014. 
[17] C. C. Gornick, S. W. Adler, B. Pederson, J. Hauck, J. Budd, and J. Schweitzer, "Validation of a new noncontact catheter system for electroanatomic mapping of left ventricular endocardium," Circulation, vol. 99, pp. 829-35, Feb 161999.

[18] A. Ahmad, J. L. Salinet, Jr., P. Brown, J. H. Tuan, P. Stafford, G. A. Ng, et al., "QRS subtraction for atrial electrograms: flat, linear and spline interpolations," Med Biol Eng Comput, vol. 49, pp. 1321-8, Nov 2011.

[19] J. L. Salinet, Jr., J. P. Madeiro, P. C. Cortez, P. J. Stafford, G. A. Ng, and F. S. Schlindwein, "Analysis of QRS-T subtraction in unipolar atrial fibrillation electrograms," Med Biol Eng Comput, vol. 51, pp. 1381-91, Dec 2013.

[20] S. G. Johnson and M. Frigo, "A modified split-radix FFT with fewer arithmetic operations," Ieee Transactions on Signal Processing, vol. 55, pp. 111-119, Jan 2007.

[21] A. H. Nuttall, "Some Windows with Very Good Sidelobe Behavior," Ieee Transactions on Acoustics Speech and Signal Processing, vol. 29, pp. 84-91, 1981.

[22] J. L. Salinet, G. N. Oliveira, F. J. Vanheusden, J. L. D. Comba, G. A. Ng, and F. S. Schlindwein, "Visualizing Intracardiac Atrial Fibrillation Electrograms Using Spectral Analysis," Computing in Science \& Engineering, vol. 15, pp. 79-87, Mar-Apr 2013.

[23] T. J. Misa, "An Interview with Edsger W. Dijkstra," Communications of the Acm, vol. 53, pp. 41-47, Aug 2010.

[24] R. H. Clayton and M. P. Nash, "Analysis of cardiac fibrillation using phase mapping," Card Electrophysiol Clin, vol. 7, pp. 49-58, Mar 2015.

[25] A. van Oosterom, "Triangulating the human torso," The Computer Journal, vol. 21, pp. 253-258, January 1, 19781978.

[26] G. Cramer, Introduction A L'Analyse Des Lignes Courbes Algébriques.: Cramer \& Philibert, 1750.

[27] J. Jalife, D. Filgueiras Rama, and O. Berenfeld, "Letter by Jalife et al Regarding Article, "Quantitative Analysis of Localized Sources Identified by Focal Impulse and Rotor Modulation Mapping in Atrial Fibrillation"," Circ Arrhythm Electrophysiol, vol. 8, pp. 1296-8, Oct 2015. 
[28] P. Benharash, E. Buch, P. Frank, M. Share, R. Tung, K. Shivkumar, et al., "Quantitative analysis of localized sources identified by focal impulse and rotor modulation mapping in atrial fibrillation," Circ Arrhythm Electrophysiol, vol. 8, pp. 554-61, Jun 2015.

[29] X. Li, J. L. Salinet, T. P. Almeida, F. J. Vanheusden, G. S. Chu, G. A. Ng, et al., "A platform to guide catheter ablation of persistent atrial fibrillation using dominant frequency mapping," presented at the Computing in Cardiology (CinC), 2014, Cambridge, MA, 2014.

[30] V. Fuster, L. E. Ryden, D. S. Cannom, H. J. Crijns, A. B. Curtis, K. A. Ellenbogen, et al., "ACC/AHA/ESC 2006 guidelines for the management of patients with atrial fibrillation executive summary - A report of the American College of Cardiology/American Heart Association Task Force on practice guidelines and the European Society of Cardiology Committee for Practice Guidelines (Writing Committee to Revise the 2001 Guidelines for the Management of Patients with Atrial Fibrillation) Developed in collaboration with the European Heart Rhythm Association and the Heart Rhythm Society," Eur Heart J, vol. 27, pp. 1979-2030, Aug 2006.

[31] S. Nattel, B. Burstein, and D. Dobrev, "Molecular Remodelling and Chronic Atrial Fibrillation," in Cardiac electrophysiology : from cell to bedside. vol. Vol. 2000, D. P. Zipes and J. Jalife, Eds., 5th ed. ed Philadelphia, Pa.: Saunders, 2009, pp. 1-13.

[32] I. Ziad, J. M. Miller, and D. P. Zipes, "Atrial Fibrillation," in Clinical Arrhythmology and Electrophysiology, I. Ziad, J. M. Miller, and D. P. Zipes, Eds., 2nd ed Philadelphia, Pa.: Saunders, 2013, pp. 290-374.

[33] S. Fichtner, K. Sparn, T. Reents, S. Ammar, V. Semmler, R. Dillier, et al., "Recurrence of paroxysmal atrial fibrillation after pulmonary vein isolation: is repeat pulmonary vein isolation enough? A prospective, randomized trial," Europace, vol. 17, pp. 1371-5, Sep 2015.

[34] M. Haissaguerre, P. Jais, D. C. Shah, A. Takahashi, M. Hocini, G. Quiniou, et al., "Spontaneous initiation of atrial fibrillation by ectopic beats originating in the pulmonary veins," N Engl J Med, vol. 339, pp. 659-66, Sep 031998. 
[35] J. Jalife, O. Berenfeld, and M. Mansour, "Mother rotors and fibrillatory conduction: a mechanism of atrial fibrillation," Cardiovasc Res, vol. 54, pp. 204-16, May 2002.

[36] M. Mansour, R. Mandapati, O. Berenfeld, J. Chen, F. H. Samie, and J. Jalife, "Left-toright gradient of atrial frequencies during acute atrial fibrillation in the isolated sheep heart," Circulation, vol. 103, pp. 2631-6, May 292001.

[37] J. W. Jarman, T. Wong, P. Kojodjojo, H. Spohr, J. E. Davies, M. Roughton, et al., "Organizational index mapping to identify focal sources during persistent atrial fibrillation," J Cardiovasc Electrophysiol, vol. 25, pp. 355-63, Apr 2014.

[38] S. M. Narayan, J. Patel, S. Mulpuru, and D. E. Krummen, "Focal impulse and rotor modulation ablation of sustaining rotors abruptly terminates persistent atrial fibrillation to sinus rhythm with elimination on follow-up: a video case study," Heart Rhythm, vol. 9, pp. 1436-9, Sep 2012.

[39] R. J. Schilling, A. H. Kadish, N. S. Peters, J. Goldberger, and D. W. Davies, "Endocardial mapping of atrial fibrillation in the human right atrium using a non-contact catheter," European Heart Journal, vol. 21, pp. 550-564, Apr 2000.

[40] M. J. Earley, D. J. Abrams, S. C. Sporton, and R. J. Schilling, "Validation of the noncontact mapping system in the left atrium during permanent atrial fibrillation and sinus rhythm," J Am Coll Cardiol, vol. 48, pp. 485-91, Aug 012006.

[41] J. W. E. Jarman, T. Wong, P. Kojodjojo, H. Spohr, J. E. Davies, M. Roughton, et al., "Spatiotemporal behavior of high dominant frequency during paroxysmal and persistent atrial fibrillation in the human left atrium," Circulation: Arrhythmia and Electrophysiology, vol. 5, pp. 650-658, 2012.

[42] R. J. Schilling, N. S. Peters, and W. Davies, "Simultaneous endocardial mapping in the human left ventricle using a noncontact catheter - Comparison of contact and reconstructed electrograms during sinus rhythm," Circulation, vol. 98, pp. 887-898, Sep 1 1998. 
[43] S. Gojraty, N. Lavi, E. Valles, S. J. Kim, J. Michele, and E. P. Gerstenfeld, "Dominant frequency mapping of atrial fibrillation: comparison of contact and noncontact approaches," J Cardiovasc Electrophysiol, vol. 20, pp. 997-1004, Sep 2009.

[44] Y. J. Lin, S. Higa, T. Kao, H. W. Tso, C. T. Tai, S. L. Chang, et al., "Validation of the frequency spectra obtained from the noncontact unipolar electrograms during atrial fibrillation," J Cardiovasc Electrophysiol, vol. 18, pp. 1147-53, Nov 2007.

[45] J. Ng and J. J. Goldberger, "Understanding and interpreting dominant frequency analysis of AF electrograms," J Cardiovasc Electrophysiol, vol. 18, pp. 680-5, Jun 2007.

[46] J. Ng, A. H. Kadish, and J. J. Goldberger, "Effect of electrogram characteristics on the relationship of dominant frequency to atrial activation rate in atrial fibrillation," Heart Rhythm, vol. 3, pp. 1295-305, Nov 2006.

[47] J. Ng, A. H. Kadish, and J. J. Goldberger, "Technical considerations for dominant frequency analysis," J Cardiovasc Electrophysiol, vol. 18, pp. 757-64, Jul 2007.

[48] M. K. Stiles, A. G. Brooks, B. John, Shashidhar, L. Wilson, P. Kuklik, et al., "The effect of electrogram duration on quantification of complex fractionated atrial electrograms and dominant frequency," J Cardiovasc Electrophysiol, vol. 19, pp. 252-8, Mar 2008.

[49] F. J. Harris, "On the use of windows for harmonic analysis with the discrete Fourier transform," Proceedings of the IEEE, vol. 66, pp. 51-83, 1978.

[50] F. Gaita, R. Riccardi, L. Calo, M. Scaglione, L. Garberoglio, R. Antolini, et al., "Atrial mapping and radiofrequency catheter ablation in patients with idiopathic atrial fibrillation. Electrophysiological findings and ablation results," Circulation, vol. 97, pp. 2136-45, Jun 021998.

[51] N. Dastagir, F. J. Vanheusden, G. S. Chu, J. L. Salinet, X. Li, T. P. Almeida, et al., "Spatiotemporal Analysis of Phase and Frequency Dynamics in Human Persistent Atrial Fibrillation," Circulation, vol. 132, p. A15890, November 10, 20152015.

[52] T. G. Oesterlein, J. Schmid, S. Bauer, A. Jadidi, C. Schmitt, O. Dossel, et al., "Analysis and visualization of intracardiac electrograms in diagnosis and research: Concept and 
application of KaPAVIE," Comput Methods Programs Biomed, vol. 127, pp. 165-73, Apr 2016.

[53] N. S. Dastagir, J.; Vanheusden, F.J.; Almeida, T.P.; Xin Li; Chu, G.S.; Ng, G.A.; Schlindwein, F.S., "Spatiotemporal behaviour of high dominant frequency during persistent atrial fibrillation," presented at the Computing in Cardiology (CinC), 2014, Cambridge, MA, 2014.

[54] X. Li, G. S. Chu, T. P. Almeida, F. J. Vanheusden, N. Dastagir, J. L. Salinet, et al., "Investigation on Recurrent High Dominant Frequency Spatiotemporal Patterns during Persistent Atrial Fibrillation," presented at the Computing in Cardiology Conference (CinC), 2015, Nice, France, 2015.

[55] T. P. Almeida, G. S. Chu, J. L. Salinet, F. J. Vanheusden, X. Li, J. H. Tuan, et al., "Minimizing discordances in automated classification of fractionated electrograms in human persistent atrial fibrillation," Med Biol Eng Comput, vol. 54, pp. 1695-1706, Nov 2016.

[56] H. Chubb, G. S. Chu, N. A. Qureshi, J. H. P. Gamble, S. M. A. Sohaib, R. J. Taylor, et al., "Young Investigators Competition1Left ventricular lead position, mechanical activation and myocardial scar in relation to the clinical outcome of cardiac resynchronisation therapy: the role of feature-tracking and contrast-enhanced cardiovascular magnetic resonance2Does the haemodynamic improvement of biventricular pacing truly arise from cardiac resynchronisation? quantifying the contribution of av and vv adjustment3Differential relationship of electrical delay with endocardial and epicardial left ventricular leads for cardiac resynchronisation therapy4Characterisation of the persistent af substrate through the assessment of electrophysiologic parameters in the organised vs. disorganised rhythm5Targeting cyclical highest dominant frequency in the ablation of persistent atrial fibrillation6Feasibility of fully mr-guided ablation with active tracking: from pre-clinical to clinical application," Europace, vol. 17, pp. v1-v2, 2015-10-01 00:00:00 2016. 